\title{
Stereochemical Criteria for Polypeptides and Proteins. V. Conformation of a System of Three Linked Peptide Units*
}

\author{
C. M. VENKATACHALAM, Centre of Advanced Study in Biophysics, \\ University of Madras, Madras, India and Biophysics Research Division, \\ University of Michigan, Ann Arbor, Michigan, U.S.A.
}

\begin{abstract}
Synopsis
The general conformations of a system of three linked peptide units are studied, and it is found that there are three types of conformations which contain NH. . . hydrogen bonding between the first and the third units. One of them is part of a $3_{10}$-helix, while the other two are nonhelical. The two nonhelical conformations are very similar, and in both the cases the peptide chain turns around, reversing the direction of progress. Such a conformation can therefore occur in the region where a polypeptide chain folds back on itself, as in the cross- $\beta$ structure. The method of representing these interesting tripeptide conformations in a $(\phi, \psi)$ map is described. Examples of such hydrogen-bonded, nonhelical conformations which occur in peptides and proteins are discussed-e.g., in cyclohexaglycyl, an open tetrapeptide Gly-L-Pro-L-Leu-Gly, and in parts of the lysozyme chain.
\end{abstract}

\section{Introduction}

Conformations of a pair of peptide units have already been studied by using contact-distance criteria and potential functions. ${ }^{1-4}$ Conformational maps in the $(\phi-\psi)$ plane have been worked out also for helical polypeptide chains., ${ }^{3,5,6}$ In this paper, we report studies on the possible general conformations of a system of three linked peptide units, which may be denoted by the symbols (1), (2), and (3). $\dagger$ In particular, it is found that those conformations of this system which are stabilized by $\mathrm{NH}$. . O hydrogen bonding, may be classified into three types, two of which are nonhelical

* Contribution No. 236 from the Centre of Advanced Study in Physics, University of Madras, India.

$\dagger$ This group of atoms $\mathrm{C}^{\alpha}\left(\mathrm{C}^{\prime} \mathrm{O}\right)(\mathrm{NH}) \mathrm{C}^{\alpha}$ is termed the peptide unit. We find it convenient to refer the $i$ th peptide unit by the symbol $(i)$ within parentheses, while, following Edsall et al., ${ }^{7}$ we use the symbol $i$ as a subscript to denote $i$ th amino acid residue. Short contacts between the $i$ th and $j$ th units are referred to as $(i) \ldots(j)$ short contacts. The hydrogen bonding between $\mathrm{NH}$ of the $i$ th residue and $O$ of the $j$ th residue is said to be of the type $i \rightarrow j$, taking the bond direction to be from donor to the acceptor atom, which is slightly different from that adopted by Edsall et al. but has general acceptance (Workshop held in Madras in January 1967). This simultaneous use of the unit and the residue is found to be necessary and the notation follows that adopted by Ramachandran and Sasisekharan. ${ }^{8}$ 


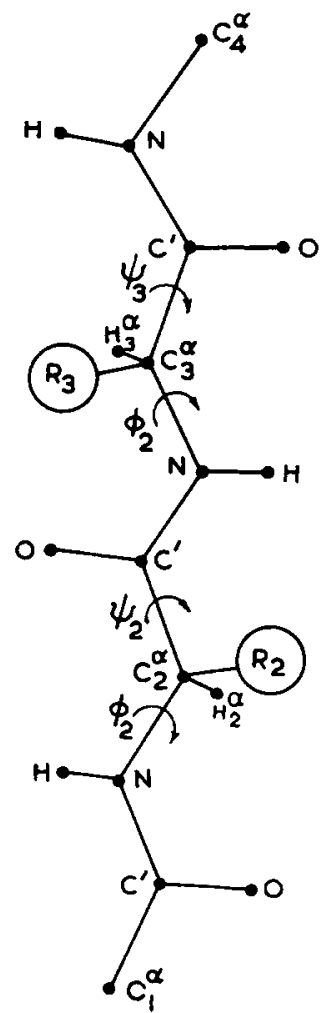

Fig. 1. Schenatic diagram of a system of three linked peptide mits. The dihedral angles $\phi_{2}, \psi_{2} ; \phi_{3}, \psi_{3}$ are marked, as well as the notation for backbone atoms.

and the third one is close to that of the $3_{10}$-helix. These predicted conformations have actually been observed in some structures, and these are discussed in relation to the theory.

Any general conformation of a system of three peptide units may be characterized by the values of the dihedral angles of rotation $\left(\phi_{2}, \psi_{2}\right)$ and $\left(\phi_{3}, \psi_{3}\right)$ at the atoms $\mathrm{C}_{2}{ }^{\alpha}$ and $\mathrm{C}_{3}{ }^{\alpha}$, respectively (Fig. 1). The factors that chiefly govern the stability of the system are the following: $(a)$ interactions between units (1) and (2) [(1) . . (2) contacts] and those between (2) and (3) $[((2) \ldots$. (3) contacts $]$; (b) hydrogen bouding between (1) and (2) and between (2) and (3); (c) interactions between units (1) and (3) [(1) . . (3) contacts]; and $(d)$ hydrogen bonding between (1) and (3).

(1) ... (2) and (2) ... (3) contacts may be made favorable by so choosing the local conformations at $\mathrm{C}_{2}{ }^{\alpha}$ and $\mathrm{C}_{3}{ }^{\alpha}$ that they lie within the allowed regions of the conformational maps for a pair of peptide units ${ }^{3}$ corresponding to the side groups $\mathrm{R}_{2}$ and $\mathrm{R}_{3}$ at $\mathrm{C}_{2}{ }^{\alpha}$ and $\mathrm{C}_{3}{ }^{\alpha}$. The factor (b) has already been studied, ${ }^{3}$ and it has been shown that the hydrogen bonding between two adjacent units may only be of the type $3 \rightarrow 1$ which is possible for a small range of conformations near $\left(95^{\circ}, 230^{\circ}\right)$. Factors $(c)$ and $(d)$ are discussed in this paper. 


\section{Details of the Calculations}

Computer programs to generate polypeptide chains have been developed in this laboratory based on the mathematical procedures already described in detail. ${ }^{3,8}$ Different conformations of the three linked peptide units were generated by varying the angles $\phi_{2}, \psi_{2}, \phi_{3}$, and $\psi_{3}$ at intervals of $10^{\circ}$. The values of these angles were chosen such that the local conformations at $\mathrm{C}_{2}{ }^{\alpha}$ and $\mathrm{C}_{3}{ }^{\alpha}$ were allowed for a pair of peptide units with glycyl alphacarbon atoms. The angle $\tau$ between the bonds $\mathrm{N}-\mathrm{C}^{\alpha}$ and $\mathrm{C}^{\alpha}-\mathrm{C}^{\prime}$ was taken to be $110^{\circ}$. The conformations considered were in general nonhelical, since the local conformations at $\mathrm{C}_{2}{ }^{\alpha}$ and $\mathrm{C}_{3}{ }^{\alpha}$ were different. A hydrogen bond search was performed for each of the conformations so developed to find out whether a hydrogen bond of the type $\mathrm{N}_{2} \mathrm{H}_{2} \ldots \mathrm{O}_{3}$ or $\mathrm{N}_{4} \mathrm{H}_{4} \ldots \mathrm{O}_{1}$ could be formed. The criteria for hydrogen bonding employed here was the same as those used for polypeptide helices $:^{3}$ the distance $N$. . O was required to be between 2.6 and $3.2 \AA$ and the angle NH-NO to be less than $30^{\circ}$. Such relaxed limits facilitated the analysis of the conformations. The hydrogen bonded conformations were subsequently scrutinized for the presence of short contacts on the basis of contact-distance criteria that were found to be quite successful with other systems. ${ }^{3,8}$ Thus a list of allowed conformations which are stabilized by $\mathrm{NH} \ldots \mathrm{O}$ hydrogen bonds between the units (1) and (3) was arrived at.

\section{Hydrogen-Bonded Conformations}

Table I lists the allowed conformations of the system of three linked peptide units which contain NH . . O hydrogen bonds between units (1) and (3); the hydrogen bond length $\mathrm{N} \ldots \mathrm{O}$, the angles $\mathrm{NH}-\mathrm{NO}$ and $\mathrm{NO}-\mathrm{CO}$ and the value of the end-to-end distance $\mathrm{C}_{1}{ }^{\alpha} \ldots \mathrm{C}_{4}{ }^{\alpha}$ are given in the table. (Calculations have been performed by varying the dihedral angles $\phi$ and $\psi$ at intervals of $10^{\circ}$. The results are, however, given in Table I only at intervals of $30^{\circ}$ and detailed tables will be reported elsewhere..$^{9,10}$ The table covers only one half of the data, and the hydrogen bonding parameters and the distance $\mathrm{C}_{1}{ }^{\alpha} \ldots \mathrm{C}_{4}{ }^{\alpha}$ for a given $\left(\phi_{2}, \psi_{2}, \phi_{3}, \psi_{3}\right)$ are identical to those for the "inverse conformations" $\left(-\phi_{2},-\psi_{2} ;-\phi_{3},-\psi_{3}\right)$, since they both pertain only to the backbone and are independent of whether the residues are $\mathrm{L}$ or $\mathrm{D}$.)

It is interesting to note that all hydrogen bonds are of the type $\mathrm{N}_{4} \mathrm{H}_{4} \ldots \mathrm{O}_{1}$ (type $4 \rightarrow 1$ ), i.e., in the reverse direction from the third unit to the first unit and the type $\mathrm{N}_{2} \mathrm{H}_{2} \ldots \mathrm{O}_{3}(1 \rightarrow 2$ type, from the first unit to the third unit) is not possible for allowed conformations.

A study of the Table I yields many interesting points. First, for hydrogen bond formation ( of the type $4 \rightarrow 1$ ), it may be seen that the value of $\phi_{2}$ should lie between $110^{\circ}$ and $130^{\circ}$ (or between $250^{\circ}$ and $230^{\circ}$ ). lurther, neither $\left(\phi_{2}, \psi_{2}\right)$ nor $\left(\phi_{3}, \psi_{3}\right)$ occurs in the region near $\left(95^{\circ}, 230^{\circ}\right)$ (or its inverse conformation $\left(265^{\circ}, 130^{\circ}\right)$. It follows, therefore, that it is not possible to form simultaneously both $3 \rightarrow 1$ and $4 \rightarrow 1$ types hydrogen bonding. 
TABI,E I

List of Allowed and Hydrogen Bonded Conformations of a system of Three Linked Peptide Units in Intervals of $30^{\circ}$ for the Dihedral Angles ${ }^{\mathrm{a}}$

\begin{tabular}{|c|c|c|c|c|c|c|c|c|}
\hline No. & $\phi_{2}$ & $\psi_{2}$ & $\phi_{3}$ & $\psi_{3}$ & $\mathrm{~N}_{4} \ldots \mathrm{O}_{\mathrm{i}}$ & $\begin{array}{c}\text { Angle } \\
\mathrm{NH}-\mathrm{NO}\end{array}$ & $\begin{array}{c}\text { Angle } \\
\mathrm{NO}-\mathrm{CO}\end{array}$ & $\mathrm{C}_{1}^{\alpha} \ldots \mathrm{C}_{1}^{\alpha}$, \\
\hline 1 & $120^{\circ}$ & $150^{\circ}$ & $60^{\circ}$ & $210^{\circ}$ & 2.99 & $16^{\circ}$ & $79^{\circ}$ & 4.37 \\
\hline 2 & $120^{\circ}$ & $150^{\circ}$ & $90^{\circ}$ & $180^{\circ}$ & 2.72 & $21^{\circ}$ & $70^{\circ}$ & 4.64 \\
\hline 3 & $120^{\circ}$ & $150^{\circ}$ & $120^{\circ}$ & $150^{\circ}$ & 2.90 & $23^{\circ}$ & $62^{\circ}$ & 5.39 \\
\hline 4 & $120^{\circ}$ & $150^{\circ}$ & $120^{\circ}$ & $180^{\circ}$ & 2.94 & $28^{\circ}$ & $59^{\circ}$ & 5.62 \\
\hline 5 & $120^{\circ}$ & $180^{\circ}$ & $90^{\circ}$ & $150^{\circ}$ & 2.71 & $18^{\circ}$ & $49^{\circ}$ & 5.44 \\
\hline 6 & $120^{\circ}$ & $180^{\circ}$ & $90^{\circ}$ & $180^{\circ}$ & 2.73 & $23^{\circ}$ & $45^{\circ}$ & 5.67 \\
\hline 7 & $120^{\circ}$ & $180^{\circ}$ & $120^{\circ}$ & $150^{\circ}$ & 3.15 & $6^{\circ}$ & $47^{\circ}$ & 6.40 \\
\hline 8 & $120^{\circ}$ & $270^{\circ}$ & $240^{\circ}$ & $210^{\circ}$ & 3.12 & $5^{\circ}$ & $79^{\circ}$ & 4.64 \\
\hline 9 & $120^{\circ}$ & $270^{\circ}$ & $270^{\circ}$ & $180^{\circ}$ & 2.71 & $22^{\circ}$ & $70^{\circ}$ & 4.29 \\
\hline 10 & $120^{\circ}$ & $270^{\circ}$ & $270^{\circ}$ & $210^{\circ}$ & 2.70 & $19^{\circ}$ & $57^{\circ}$ & 4.52 \\
\hline 11 & $120^{\circ}$ & $270^{\circ}$ & $300^{\circ}$ & $180^{\circ}$ & 2.51 & $11^{\circ}$ & $43^{\circ}$ & 4.69 \\
\hline 12 & $120^{\circ}$ & $270^{\circ}$ & $330^{\circ}$ & $150^{\circ}$ & 2.77 & $13^{\circ}$ & $31^{\circ}$ & 5.43 \\
\hline 13 & $120^{\circ}$ & $300^{\circ}$ & $240^{\circ}$ & $210^{\circ}$ & 2.91 & $24^{\circ}$ & $57^{\circ}$ & 4.81 \\
\hline 14 & $120^{\circ}$ & $300^{\circ}$ & $270^{\circ}$ & $180^{\circ}$ & 2.74 & $21^{\circ}$ & $45^{\circ}$ & 4.96 \\
\hline 15 & $120^{\circ}$ & $300^{\circ}$ & $300^{\circ}$ & $150^{\circ}$ & 3.02 & $15^{\circ}$ & $35^{\circ}$ & 5.70 \\
\hline
\end{tabular}

a The hydrogen bond data are identical for the corresponding inverse conformations given by $\left(-\phi_{2},-\psi_{2}\right),\left(-\phi_{3},-\psi_{3}\right)$.

There are three broad regions where $4 \rightarrow 1$ hydrogen bonded conformations between the last and the first of the three peptide units occur. These are, $(a)$ around $\left(120^{\circ}, 150^{\circ} ; 90^{\circ}, 180^{\circ}\right)$ (type I), (b) near $\left(120^{\circ}, 300^{\circ} ; 260^{\circ}\right.$, $\left.180^{\circ}\right)$ (type II), and (c) close to $\left(120^{\circ}, 150^{\circ} ; 120^{\circ}, 150^{\circ}\right)$ (type III). The conformation of type III is helical and is easily recognized to be a part of a $33_{10}$-helixlike structure. Since the present calculations deal with general conformations, the helical conformations are naturally included in them. In fact, conformation I may be obtained by modifying the helical conformation III with a variation of the local conformation at $\mathrm{C}_{3}{ }^{\alpha}$. The conformations of the types I and II are clearly nonhelical, but they are interesting and are closely similar to each other. In both these conformations, the peptide chain turns around reversing the direction of progress. Schematic diagrams of these are given in ligure 2. The four $\alpha$-carbon atoms lie roughly in a plane which contains also the hydrogen bond $\mathrm{N}_{4} \mathrm{H}_{4} \ldots \mathrm{O}_{1}$. For both these conformations, the disposition of the peptide units (1) and (3) are very similar, the planes of these units being coplanar with the horizontal plane (i.e., the plane containing the $\mathrm{C}^{\alpha}$ atoms). The second peptide unit is roughly perpendicular to this horizontal plane, with $\mathrm{C}=\mathrm{O}$ pointing in one direction in $\mathrm{I}$ and in the opposite direction in II. Thus, the difference between I and II is only in that the second unit is flipped over in one with respect to the other conformation. This "flip over" of course, changes the local conformations at $\mathrm{C}_{2}{ }^{\alpha}$ and $\mathrm{C}_{3}{ }^{\alpha}$ but preserves the $4 \rightarrow 1$ hydrogen bond. 


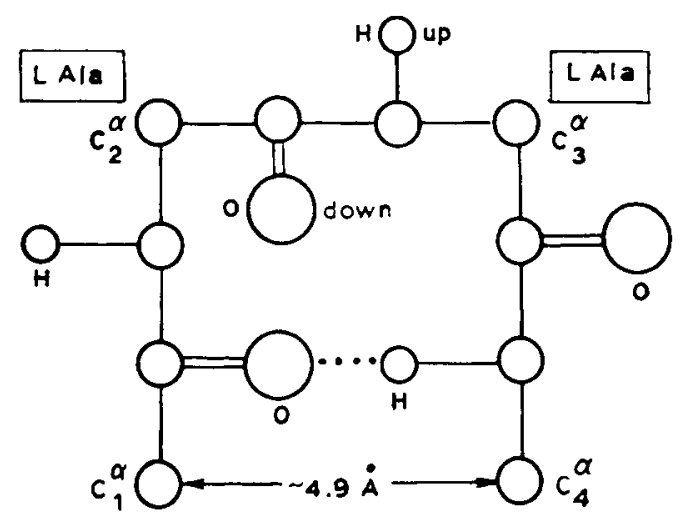

A

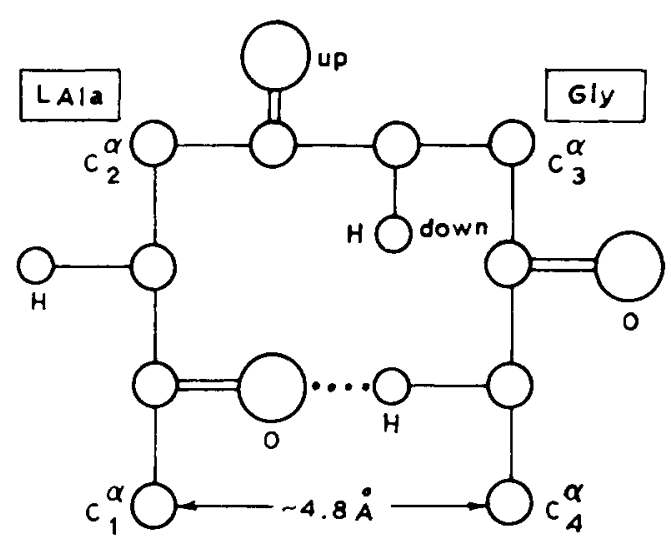

B

Fig. 2. The two possible conformations of three peptide units which lead to chain reversal and have $4 \rightarrow 1$ hydrogen bonding. (A) conformation of type $I ;(B)$ conformation of type II.

It may be noted that the inverse conformations of the above three conformations I, II, and III are also possible, and their properties are quite similar to those already discussed. In fact, these conformations are the mirror images of the conformations, I, II, and III and may be denoted by $\mathrm{I}^{\prime}, \mathrm{II}^{\prime}$ and $\mathrm{III}^{\prime}$, respectively. Thus, III' corresponds to the left-handed $3_{10}$-helix, and I' and $\mathrm{II}^{\prime}$ have the middle peptide unit related by a "flipover."

\section{Representation of Tripeptide Conformations in the $(\phi-\psi)$ Map}

We may use the following method to represent any general conformation of a system of three peptide units in the conformational map. Since a point in the $(\phi, \psi)$ plane denotes a local conformation at a single $\alpha$-carbon 


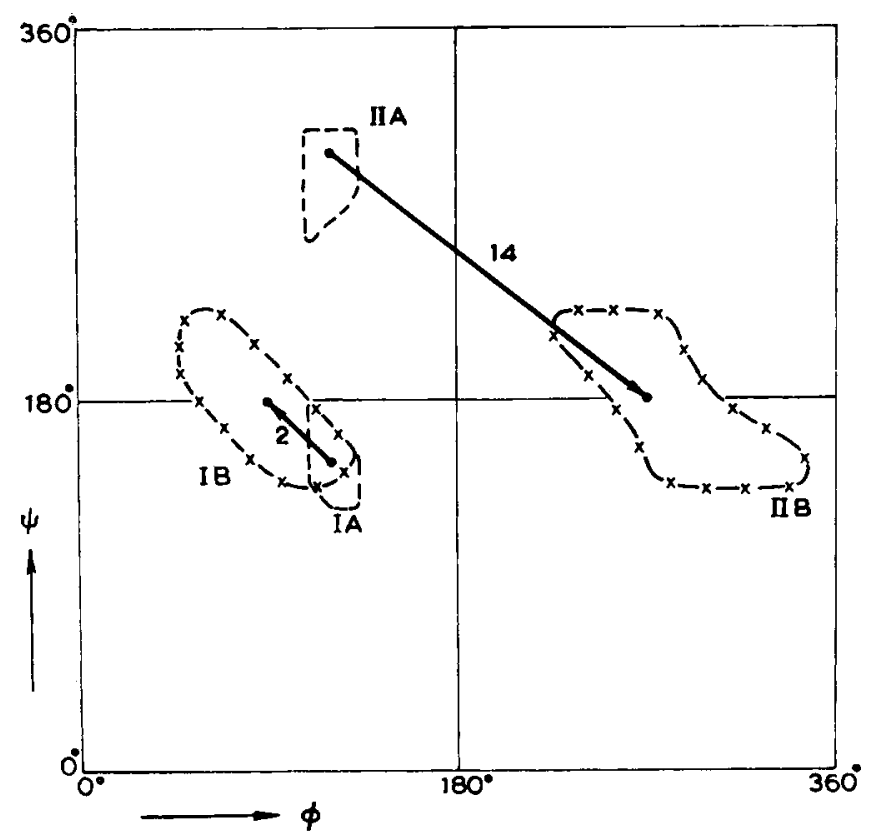

(a)

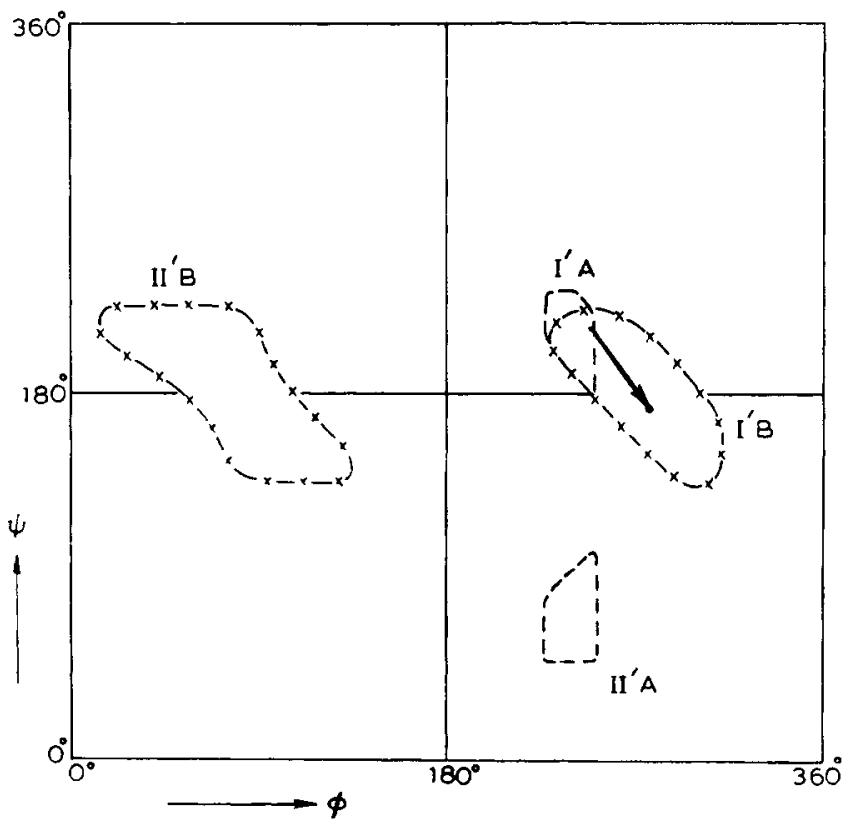

(b)

Fig. 3. Conformational maps. (a) Conformational map showing regions of allowed hydrogen bonded conformations of the types I and II. The $4 \rightarrow 1$ hydrogen bonded tripeptide conformations are represented by a vector starting from a point in a region $A$ bounded by line (--) and ending in the corresponding region $B$ bounded by line $(-\times-X)$. Two typical vectors corresponding to the conformations I and II (nos. 2 and 14 from Table I) are shown. (b) Conformational map similar to Fig. $3 a$ for conformations I' and $\mathrm{II}^{\prime}$, which are mirror images of I and II respectively. The vector marked denotes the conformation of type $I^{\prime}$ observed in cyclohexaglycyl. 
atom, a vector starting from $\left(\phi_{2}, \psi_{2}\right)$ and ending in $\left(\phi_{3}, \psi_{3}\right)$ will indicate a tripeptide conformation in the $(\phi, \psi)$ plane. For $4 \rightarrow 1$ hydrogen bonding, the two points connected by this vector should lie within certain regions in the conformational map. These regions are shown in Figure 3 for conformations I and II. (As mentioned earlier, the helical conformation III may be considered to be included in the conformation I). The starting point of the vector should lie within the region bounded by the dashed line (--) and the ending point in the corresponding region bounded by the line $(-X-X)$. These regions are referred to as A and B respectively; e.g., IA and IB for conformation I and IIA and IIB for conformation II. The conformation corresponding to No. $2\left(120^{\circ}, 150^{\circ} ; 90^{\circ}, 180^{\circ}\right)$ in Table I will correspond to the vector marked 2 in Figure $3 a$ (type I) and that corresponding to No. $14\left(120^{\circ}, 300^{\circ} ; 270^{\circ}, 180^{\circ}\right)$ to a vector marked 14 in the same figure (type II). The latter will be seen to join a point in region IIA to another point in region IIB.

The restrictions on the points connected by a vector, shown in Figure 3 form only the necessary set of conditions, but these are not sufficient. This is because the extent of variation in $\left(\phi_{3}, \psi_{3}\right)$ depends on the value of $\left(\phi_{2}, \psi_{2}\right)$, or vice versa. Therefore, all vectors running from points in a region $\mathrm{A}$ to any point in the corresponding region $\mathrm{B}$ do not necessarily represent hydrogen bonded conformations. However, the hydrogen bonded conformations will be contained within the set of such vectors from $\mathrm{A}$ to $\mathrm{B}$. Figure $3 a$ covers only one half of the possible conformations, and the other half consisting of the inverse conformations $\mathrm{I}^{\prime}$ and $\mathrm{II}^{\prime}$ is shown in Figure $3 b$. A typical vector observed in the cyclohexaglycine structure is shown in Figure $3 b$.

\section{Side Groups at $\mathrm{C}_{2}{ }^{\alpha}$ and $\mathrm{C}_{3}{ }^{\alpha}$}

From the local conformations at $\mathrm{C}_{2}{ }^{\alpha}$ and $\mathrm{C}_{3}{ }^{\alpha}$, it is possible to determine, to a certain extent, the side groups that may be accommodated at $\mathrm{C}_{2}{ }^{\alpha}$ and $\mathrm{C}_{3}{ }^{\alpha}$ without rupture of the $4 \rightarrow 1$ hydrogen bonding. The results are summarized in Table II.

TABLE II

Amino Acid Residues That Can Occur at $\mathrm{C}_{2}{ }^{\alpha}$ and $\mathrm{C}_{3}{ }^{\alpha}$ of the System of Three Linked Peptide Units for the $4 \rightarrow 1$ Hydrogen-Bonded Conformations; I' and II' are the Inverse Conformations of I and II, Respectively a,b

\begin{tabular}{clll}
\hline I & \multicolumn{1}{c}{$\mathrm{I}^{\prime}$} & \multicolumn{1}{c}{ II } & \multicolumn{1}{c}{ II $^{\prime}$} \\
\hline L-Ala L-Ala & Gly-Gly & L-Ala-Gly & Gly-L-Ala \\
& D-Ala-D-Ala & L-Ala-D-Ala & D-Ala-L-Ala \\
L-Pro L-Ala & L-Pro-Gly & Gly-L-Pro \\
& D-Pro-D-Ala & & D-Pro-L-Ala \\
& D-Pro-Gly & & \\
\hline
\end{tabular}

a Wherever L-Ala can occur, larger side groups in L-configuration may also be accommodated.

$\mathrm{b}$ The cases where the proline side chain can be accommodated are shown separately in the second half of the table. 
By plotting the local conformations at $\mathrm{C}_{2}{ }^{\alpha}$ and $\mathrm{C}_{3}{ }^{\alpha}$ on glycyl and alanyl (1) . . (2) conformational maps ${ }^{2,3}$ it may be seen that an L-alanyl group can be quite readily accommodated at $\mathrm{C}_{2}{ }^{\alpha}$ for both the conformations $\mathrm{I}$ and II, and at $\mathrm{C}_{3}{ }^{\alpha}$ for the conformation $\mathrm{I}$. It may, however, be seen from Figure $3 a$ that only a very small portion close to $\left(240^{\circ}, 210^{\circ}\right)$ of the region IIB falls within the allowed region of alanyl (1) . . (2) conformational map. Therefore, one may expect that in most cases only a glycyl residue can readily occur at $\mathrm{C}_{3}{ }^{\alpha}$ for conformation II.

Even though for conformational type II the local conformation around $\left(260^{\circ}, 180^{\circ}\right)$ at $\mathrm{C}_{3}{ }^{\alpha}$ cannot readily accommodate an L-alanyl group, it can however, quite readily accept a D-alanyl group. (This may be seen by inverting the $(\phi, \psi)$ map of Ramachandran et al. $\left.{ }^{1-3}\right)$. Thus, in the top half of the Table II, the conformational type II can accommodate the sequence either L-Ala-Gly or L-Ala-D-Ala.

The possibility of having larger side groups at the $\mathrm{C}^{\alpha}$ atom, where an alanyl group can readily occur, has also been studied. Since $\phi_{2} \sim 120^{\circ}$ for both conformations I and II, this immediately suggests the possibility of having prolyl and hydroxyprolyl side groups at $\mathrm{C}_{2}{ }^{\alpha}$. Model building clearly shows that this is indeed possible. It is also found that if alanine can occur at an $\alpha$-carbon atom, then larger side groups may also be accommodated at the $\mathrm{C}^{\alpha}$ atom, if the conformation of the side groups is taken care of properly. Table II summarizes the types of side groups that can be accommodated at $\mathrm{C}_{2}{ }^{\alpha}$ and $\mathrm{C}_{3}{ }^{\alpha}$. Larger side groups may, however, reduce the extent of flexibility of the hydrogen bonded system, but this aspect has not been studied in detail. The most interesting observations are $(a)$ if the conformational type II occurs in a protein or polypeptide chain with only L-residues, it must have, in general, Gly at $\mathrm{C}_{3}{ }^{\alpha} ;(b)$ for a similar chain with only L-residues, conformational type $\mathrm{I}^{\prime}$ can occur readily only for a sequence Gly-Gly.

\section{Comparison with Experimental Observations}

There is, indeed, experimental evidence for the occurrence of conformations of the types I and II in cyclic peptides whose structures have been determined by x-ray methods. These are listed in Table III where the conformations observed in the peptides have been compared with the nearest conformation from the compiled list.

In cyclohexaglycyl, ${ }^{11}$ conformations $\mathrm{I}$ and $\mathrm{I}^{\prime}$ are found to occur. A molecule of this cyclic hexapeptide is shown in Figure 4 and the values of $(\phi, \psi)$ at each $\alpha$-carbon atom are marked. The set of three peptide units from $\mathrm{C}_{1}{ }^{\alpha}$ to $\mathrm{C}_{4}{ }^{\alpha}$ (via $\mathrm{C}_{2}{ }^{\alpha}$ and $\mathrm{C}_{3}{ }^{\alpha}$ ) have conformation $\mathrm{I}$ and those from $\mathrm{C}_{4}{ }^{\alpha}$ and $\mathrm{C}_{1}{ }^{\alpha}$ (via $\mathrm{C}_{5}{ }^{\alpha}$ and $\mathrm{C}_{6}{ }^{\alpha}$ ) also have the conformation I. Since glycine is not asymmetric, there is another molecule in the structure, which is the mirror image of the above example and this has two sets of three peptide units having the conformation $\mathrm{I}^{\prime}$.

On the other hand, only on example of conformation II occurs in the crystal structure Ferrichrome $A^{12,13}$ which has only L-amino acids and is 


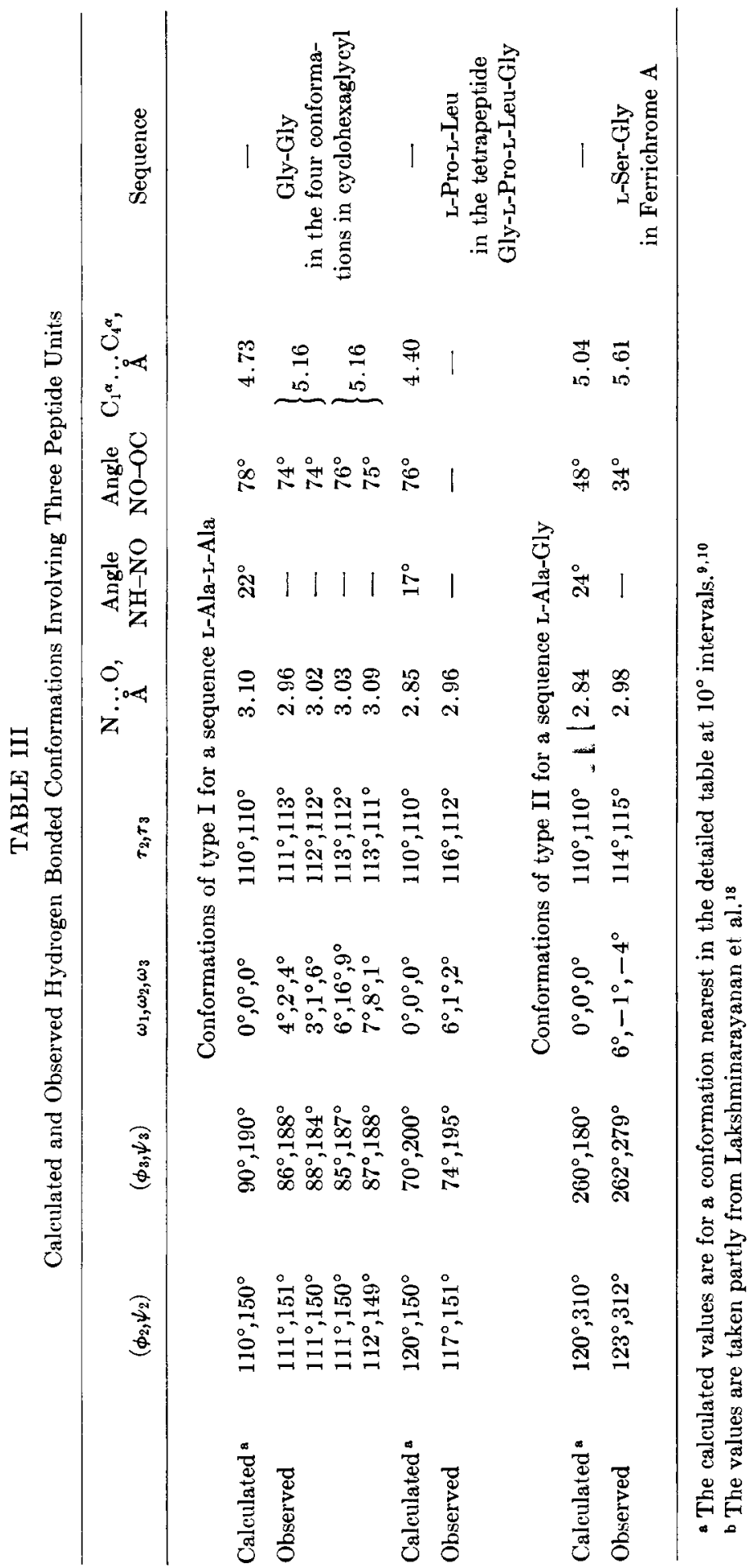




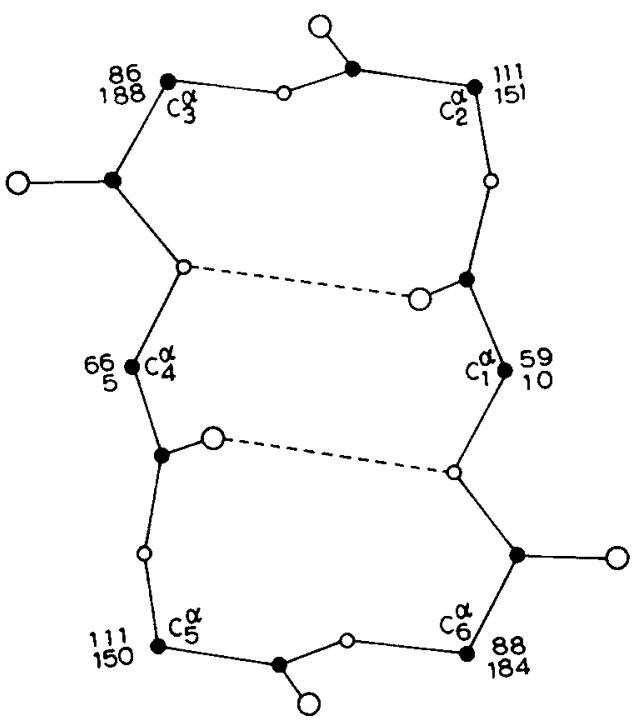

Fig. 4. Projection of a molecule of cyclohexaglycyl hemihydrate whose structure has been determined by $\mathrm{x}$-ray methods. ${ }^{11}$

noncentrosymmetric. In this case, it is interesting that the sequence observed is L-Ser-Gly which agrees well with the permitted sequence for conformation II, with the second one being a glycyl residue.

Recently, the crystal structure of a tetrapeptide, $p$-bromocarbobenzoxyglycyl-L-prolyl-L-leucylglycine has been accurately solved by x-ray methods by Kakudo and co-workers. ${ }^{14,15}$ In this structure also, the peptide chain is folded back at the prolyl and leucyl residues so that there is a hydrogen bond between the $\mathrm{NH}$ group of the last glycyl residue and the oxygen of the first glycyl residue (type $4 \rightarrow 1$ ). The sequence at $\mathrm{C}_{2}{ }^{\alpha}$ and $\mathrm{C}_{3}{ }^{\alpha}$ in this structure is L-Pro-L-Leu, which would allow only conformation I, but not II, and the conformation I is actually observed.

Hydrogen bonded conformations of the type $4 \rightarrow 1$ are found to occur in the nonhelical regions of the enzyme lysozyme. Though the information regarding the hydrogen bonding schemes in this protein is not available in the published papers, ${ }^{16,17}$ some inferences could be made from the data on dihedral angles $(\phi, \psi)$ in the polypeptide chain. Those tripeptide conformations occurring in the chain of lysozyme which fall within the region shown in Figure 3 and are suitable for forming $\mathrm{NH} \ldots \mathrm{O}$ hydrogen bonding, are listed in the upper portion of Table IV. These are evidently nonhelical conformations stabilized by $\mathrm{NH}$. . . O hydrogen bonds of the type $4 \rightarrow 1$. In addition, the conformations listed in the lower part of Table IV fall slightly outside, with $\phi, \psi$ deviating about by $10^{\circ}$ from the limits of the regions marked in Figure 3a. Nevertheless, they may also possibly represent $4 \rightarrow 1$ hydrogen bonded conformations, since the variations in the dihedral angles may be due to other effects like variation in the 
TABLE IV

Nonhelical Conformations in Lysozyme Which Contain a NH...O

Hydrogen Bond of the Type $4 \rightarrow 1$

\begin{tabular}{ccccccc}
\hline $\begin{array}{c}\text { Residue } \\
\text { numbers }\end{array}$ & $\begin{array}{c}\text { Sequence } \\
-\mathrm{R}_{2}-\mathrm{R}_{3}-\end{array}$ & $\phi_{2}$ & $\psi_{2}$ & $\phi_{3}$ & $\psi_{3}$ & $\begin{array}{c}\text { Confor- } \\
\text { mational } \\
\text { type }\end{array}$ \\
\hline \multicolumn{7}{c}{ Within the allowed regions } \\
$55-56$ & Ileu-Leu & $134^{\circ}$ & $145^{\circ}$ & $72^{\circ}$ & $190^{\circ}$ & I \\
$96-97$ & Lys-Lys & $117^{\circ}$ & $129^{\circ}$ & $105^{\circ}$ & $162^{\circ}$ & I \\
$116-117$ & Lys-Gly & $127^{\circ}$ & $285^{\circ}$ & $276^{\circ}$ & $202^{\circ}$ & II \\
$123-124$ & Try-Ileu & $116^{\circ}$ & $142^{\circ}$ & $83^{\circ}$ & $176^{\circ}$ & I \\
$125-126$ & Arg-Gly & $110^{\circ}$ & $309^{\circ}$ & $279^{\circ}$ & $168^{\circ}$ & II \\
\multicolumn{7}{c}{} \\
$9-10$ & Slightly outside the allowed regions & & \\
$33-34$ & Ala-Ala & $126^{\circ}$ & $130^{\circ}$ & $115^{\circ}$ & $140^{\circ}$ & I \\
$47-48$ & Lys-Phe & $115^{\circ}$ & $129^{\circ}$ & $108^{\circ}$ & $151^{\circ}$ & I \\
& Thr-Asn & $111^{\circ}$ & $161^{\circ}$ & $87^{\circ}$ & $217^{\circ}$ & I \\
\hline
\end{tabular}

peptide geometry and bond angles, which have not been taken into account in the present study.

An example of $4 \rightarrow 1$ hydrogen bonded conformation is observed also in the nonhelical region of the myoglobin structure at residues 44-45. This has a sequence Asp-Arg, which is allowed for this. So also, the conformation at 79-80 lies slightly outside the region for conformation $I^{\prime}$ and presumably it may contain $4 \rightarrow 1$ hydrogen bonding. This has a sequence Lys-Gly, and a slight extension of $I^{\prime}$ can accommodate $I_{A y s}$ in the first position, but the second one has to be Gly, as observed.*

It is interesting that, for the two instances of conformation II in lysozyme the residue containing $\mathrm{C}_{3}{ }^{\alpha}$ is glycine, again in agreement with the conformational analysis.

It is clear that the diagrams given in Figure 3 can be very useful for studying the $4 \rightarrow 1$ hydrogen bonded conformations in polypeptides and proteins.

The distance between $\mathrm{C}_{1}{ }^{\alpha}$ and $\mathrm{C}_{4}{ }^{\alpha}$ is found to be near about $4.8 \AA$ for the conformations I and II. This is interesting since, in such a case, the peptide units (1) and (3) could both be extended in either direction to form an antiparallel pleated sheet, thereby forming the basis of the mechanism for the folding back of the chain in a cross- $\beta$ structure. That is, the system of three peptide units may be taken as a "block," and such blocks may then serve as excellent places for folding back in a polypeptide chain. A particular case of this is when two such blocks get fused together, thereby cyclizing the chain. This is exactly what happens in cyclohexaglycyl (Fig. 4).

* The data on dihedral angles were kindly provided by Dr. J. C. Kendrew to Jr. G. N. Ramachandran and the results of a more detailed study of this will be published, after permission is obtained from the original investigators. 
The author would like to thank Professor G. N. Ramachandran for guidance and for his criticisms and useful suggestions. This investigation was partly supported by the U.S. Public Health Service Grant No. AM-10905. A part of this work was done while the author was at the University of Michigan, Ann Arbor, U.S.A. and it is a pleasure to thank Professor J. L. Oncley for his encouragement and hospitality.

\section{References}

1. G. N. Ramachandran, C. Ramakrishnan, and V. Sasisekharan, J. Mol. Biol., 7, 95 (1963).

2. C. Ramakrishnan and G. N. Ramachandran, Biophys. J., 5, 909 (1965).

3. G. N. Ramachandran, C. M. Venkatachalam, and S. Krimm, Biophys. J., 6, 849 (1966).

4. R. A. Scott and H. A. Scheraga, J. Chem. Phys., 45, 2091 (1965).

5. D. A. Brant and P. J. Flory, J. Amer. Chem. Soc., 87, 2791 (1965).

6. C. M. Venkatachalam and G. N. Ramachandran, in Conformation of Biopolymers, G. N. Ramachandran, Ed., Vol. 1, Academic Press, London, 1967, p. 83.

7. J. T. Edsall, P. J. Flory, J. C. Kendrew, A. M. Liquori, G. Nemethy, G. N. Ramachandran, and H. A. Scheraga, J. Mol. Biol., 15, 399 (1966).

8. G. N. Ramachandran and V. Sasisekharan, Adv. Protein Chem., 23, in press.

9. C. M. Venkatachalam, Ph.D. Thesis, University of Madras, Madras, 1968.

10. C. M. Venkatachalam, in Conformation of Biological Macromolecules, G. N. Ramachandran, Ed., Vol. 2, (1968) in preparation.

11. I. L. Karle and J. Karle, Acta Cryst., 16, 969 (1963).

12. A. Zalkin, J. D. Forrester, and D. H. Templeton, Science, 146, 261 (1964).

13. A. Zalkin, J. D. Forrester, and D. H. Templeton, J. Amer. Chem. Soc., 88, 810 (1966).

14. M. Kakudo, Y. Sasada, Y. Katsube, S. Sakakibara, and S. Akabori, in Aspects of Protein Structure, G. N. Ramachandran, Ed., Academic Press, London, 1963, p. 69.

15. T. Ueki, T. Ashida, M. Kakudo, Y. Sasada, and Y. Katsube, Nature, in press.

16. C. C. F. Blake, D. F. Koenig, G. A. Mair, A. C. T. North, D. C. Phillips, and V. R. Sarma, Nature, 206, 757 (1965).

17. C. C. F. Blake, G. A. Mair, A. C. T. North, D. C. Phillips, and V. R. Sarma, Proc. Roy. Soc. (London), B167, 365 (1967).

18. A. V. Lakshminarayanan, V. Sasisekharan, and G. N. Ramachandran, in Conformation of Biopolymers, Vol. 1, G. N. Ramachandran, Ed., Academic Press, London, p. 61 .

\section{Received February 13, 1968}

